\title{
Speaking order predicts memory conformity after accounting for exposure to misinformation
}

\author{
Lauren Y. Hewitt • Robert Kane • Maryanne Garry
}

Published online: 6 February 2013

(C) Psychonomic Society, Inc. 2013

\begin{abstract}
When people discuss their experiences, they can later report seeing things that they never saw, simply because they heard about those things in the discussion. One factor that may contribute to this effect is the order in which people speak; some research has investigated this issue, but it remains unclear whether a relationship exists between memory conformity and speaking order. We explored this question using data from five previous memory conformity experiments. The results provide evidence of an association between speaking order and memory conformity, such that people who spoke first in a discussion were misled less often than people who did not. These results build on previous research by demonstrating that the association could not have been caused by differences in opportunities to be misled. We could not draw conclusions about causality from the exploratory analyses, but ruled out several simple explanations of the results, and considered a variety of social and cognitive mechanisms that might account for the association. Further investigation will be required to tease apart the possible mechanisms that underlie the relationship between speaking order and memory conformity.
\end{abstract}

Keywords Memory $\cdot$ Memory conformity

Misinformation $\cdot$ Social influence $\cdot$ Eyewitness

It is now well-established that when people discuss their past experiences together, they influence each others' memoriesa phenomenon known as memory conformity (Wright, Self, \& Justice, 2000; for a review, see Wright, Memon, Skagerberg,

L. Y. Hewitt $(\bowtie) \cdot$ R. Kane

Curtin University, Perth, Western Australia, Australia

e-mail: lauren.hewitt@curtin.edu.au

M. Garry

Victoria University of Wellington, Wellington, New Zealand
\& Gabbert, 2009). One curious finding that has received attention is that the order in which people speak during a conversation affects the likelihood that each will later report false memories. More specifically, the person who speaks first is much less likely to exhibit memory conformity than a person who speaks second or not at all (Gabbert, Memon, \& Wright, 2006). Why? We address that question in this article.

One possibility is that the apparent effects of speaking order can be explained by differences in exposure to misinformation, rather than differences in susceptibility to misinformation (Lindsay, 2007). Put another way, people who speak first may show less memory conformity not because they are less influenced by misleading suggestions, but because they are less likely to be exposed to misleading suggestions. Another possibility is that people who speak first exert more influence on people who speak second than the other way around. Indeed, when people observe a conversation, they are more influenced by the first rather than second speaker, rating the person who speaks first as being more confident and more accurate than the second speaker - even when it is clear that speaking order is not decided by the speakers themselves (Wright \& Carlucci, 2011). To date, no research (to our knowledge) has examined whether Wright and Carlucci's speaking order effects also occur when a person is actively involved in the discussion, instead of observing other speakers.

Further examination of the puzzling association between speaking order and memory conformity may contribute to a better understanding of the interpersonal source-monitoring processes underlying memory conformity (Lindsay, 2008). For instance, people might rely on an interpersonal metacognitive heuristic such as that the person who initiates a conversation is more likely to be accurate (Wright et al., 2009). If so, they should be more influenced by information provided by the first speaker than by information provided by subsequent speakers (informational influence, 
Deutsch \& Gerard, 1955). This heuristic might come about from personal experience: We are probably more likely to initiate a discussion and share information when we are confident that the information that we remember is accurate - or, conversely, that we are reluctant to initiate a discussion when we are unsure (Allan, Midjord, Martin, \& Gabbert, 2011). Research investigating this issue may also enable us to better predict when people are more and less likely to be influenced by other people's shared memory reports, perhaps identifying situations in which people will be at most and at least risk of memory conformity. This kind of information may be especially important in legal situations, in which - after people have discussed their memories — we ask them to report only what they personally witnessed.

A variety of research areas lend support to the idea that speaking order may affect memory conformity; parallels can be drawn with research on social influence, cognitive dissonance, and the effects of anchoring and primacy on persuasion.

\section{Social influence}

Many well-known studies have demonstrated the compelling effects of being exposed to other people's perceptual judgments before making one's own judgments. For instance, when pairs of people estimated how much a (stationary) point of light moved, their estimates shifted toward each other's (Sherif, 1937). Importantly, people's subsequent independent estimates tended to be consistent with their counterpart's estimates, too; in other words, the information presented earlier guided later private judgments. In another series of studies, when people tried to match the lengths of unambiguously different lines, they provided inaccurate responses, conforming to the obviously incorrect judgments of a majority group of confederates (Asch, 1952; see also Hoffman, Granhag, Kwong See, \& Loftus, 2001; Mori \& Arai, 2010). Deutsch and Gerard (1955) suggested that these effects were caused by normative influence, the pressure to fit in with the social conventions of the situation. In a discussion about a shared experience, perhaps people who are exposed to another's memory before their own would be similarly influenced and conform to the first response provided, both in the immediate situation (such as in Asch's research), and on later independent reports (as in Sherif's research).

\section{Anchoring to initial judgments}

Deustch and Gerard (1955) built on Asch's (1952) research, demonstrating that when people made their own judgments before hearing others' responses, they were much less likely to be influenced, especially when their initial judgements were public. The idea is that prior commitment creates a distinct type of normative influence, self-normative influence, whereby the normative pressure to conform to one's own initial judgment is more powerful than the normative pressure to conform to the majority decision; this pressure is even more powerful following a public commitment.

Research has also demonstrated the effects of anchoring and primacy on persuasion: Simply put, people are more likely to be influenced by information that they encounter first, rather than subsequent information (e.g., Englich, Mussweiler, \& Strack, 2005; Miller \& Campbell, 1959). In addition, people are unlikely to waver from an initial decision. They strive to maintain consistency between their attitudes and behavior, and the experience of cognitive dissonancewhen one's attitudes and behavior are not consistent with each other-is uncomfortable (Festinger \& Carlsmith, 1957; Tavris $\&$ Aronson, 2007). After people form an attitude, they are less open to information that contradicts it; avoiding or rejecting contradictory information allows people to maintain cognitive consistency and avoid dissonance. In a related vein, research has shown that after people make an initial minor commitment, it is much easier to persuade them to agree to larger related requests that continue the course of action they have already committed to (the foot-in-the-door persuasion technique; Cialdini \& Goldstein, 2004).

Taken together, these findings suggest another pathway through which the first speaker's information may have more influence than does information that is presented subsequently. Put simply, someone might hold to an initial judgment or decision for a number of reasons, even when the social pressure of the initial situation has been removed. For instance, after speaking first in a discussion, people may (a) maintain their initial judgment because of the selfnormative influence to be consistent (as Deutsch \& Gerard, 1955, suggested), (b) reject subsequent contradictory information (e.g., from another speaker or from their own memory) in an effort to avoid the unpleasant experience of cognitive dissonance (as Tavris \& Aronson, 2007, suggested), or (c) remain committed to the same response because their initial behavior has persuaded them that this course of action is appropriate (as Cialdini \& Goldstein, 2004, suggested). In each of these scenarios, the result is that the information presented by the first speaker is more likely to be reported later by both the person who spoke first and the person who did not.

\section{Baseline memory confidence and accuracy}

Research has shown that people are most likely to demonstrate memory conformity when they have inaccurate memories and when they lack confidence in their memories (Wright \& Villalba, 2012). One possible alternative explanation for an association between speaking order and memory conformity is simply that people who have better 
memories, or people who are more confident in their memories, tend to speak first and also tend to be less influenced by others' suggestions. In short, speaking order might not affect memory conformity at all.

Considered as a whole, much research suggests that when discussing a shared experience, the first speaker in a pair may be less likely to be influenced by their counterpart. We addressed this issue using data sets from five previous memory conformity experiments in which people took part in pairs. Each member of the pair unknowingly saw slightly different versions of an event; the versions differed in critical aspects. Shortly afterward, the pairs discussed the event together, and each person then completed a memory test independently. For each discussed critical detail, we examined whether each person (a) spoke first and (b) was exposed to misinformation. We then examined whether speaking order was associated either with exposure to misinformation or with memory conformity.

\section{Method}

\section{Data sets}

The five data sets came from three experiments published in journals (French, Garry, \& Mori, 2008, 2011; Garry, French, Kinzett, \& Mori, 2008) and from two experiments reported in a doctoral dissertation (French, 2008, Exps. The five experiments each involved different manipulations that are not relevant to the primary research questions presented here. $2 \mathrm{~B}$ and 5). We present a brief overview of the basic methodology for the purposes of the research questions addressed here.

\section{Participants}

In total, 400 people took part in the five experiments at Victoria University of Wellington (see Table 1). They were either undergraduate psychology students, who took part in return for course credit, or people recruited around campus, who each received a voucher for their time.
Materials and procedure

The experiments all followed the same basic three-stage procedure. In Stage 1, pairs of people watched slightly different versions of a movie using the MORI technique (Mori, 2003). They each wore polarizing glasses, allowing them to see one version of a movie while blocking the other from view (see French et al., 2008, 2011; Garry et al., 2008, for details). There were eight critical differences between the movie versions. For example, in the movies used in Experiments 3, 4, and 5, one member of the pair saw the main character steal a wallet, while the other saw him put the wallet back where he found it.

In Stage 2, after a filler task, the pairs were presented with 12 questions to discuss and answer; they were not required to agree on an answer. Four questions related to four of the critical items from the movie, and the remaining eight questions were fillers. Critical items were fully counterbalanced, appearing equally as often as the discussed and nondiscussed items.

In Stage 3, after another filler task, everyone completed a surprise 20 -item, two-alternative forced choice recognition test independently. We told people that we were testing their memory for the movie. Eight of the questions related to the eight critical details; the two alternatives provided for these questions were the correct details from each movie version. The remaining 12 questions were fillers. After answering each question, participants used a scale to rate how confident they were that their answer was correct, where $1=$ not at all confident and $5=$ very confident. In the research reported here, for each person, we focused on only the four critical items that were discussed. As Table 1 illustrates, people were not always exposed to misleading information, and when they were exposed to misleading suggestions, they were not always misled. Across all experiments, participants were exposed to misleading suggestions in $64.4 \%$ of their discussions of critical items, and they exhibited memory conformity $47.7 \%$ of the times that they were exposed to misleading suggestions.

Table 1 Data-set labels, references, sample sizes, total exposure to misinformation, and total memory conformity for each data set

\begin{tabular}{lllll}
\hline Data Set & Reference & $N$ & $\begin{array}{l}\text { Exposure to Misinformation } \\
\left(\text { Total Opportunities }{ }^{\mathrm{a}}\right)\end{array}$ & Memory Conformity (Percentage) $^{\mathrm{b}}$ \\
\hline Experiment 1 & Garry, French, Kinzett, \& Mori (2008) & 40 & $81(160 ; 50.6 \%)$ & $54(66.7 \%)$ \\
Experiment 2 & French, Garry, \& Mori (2008) & 64 & $154(256 ; 60.2 \%)$ & $87(56.5 \%)$ \\
Experiment 3 & French Garry, \& Mori (2011) & 96 & $253(384 ; 65.9 \%)$ & $119(47.0 \%)$ \\
Experiment 4 & French (2008): Exp. 2B & 40 & $106(160 ; 66.3 \%)$ & $50(47.2 \%)$ \\
Experiment 5 & French (2008): Exp. 5 & 160 & $436(640 ; 68.1 \%)$ & $181(41.5 \%)$ \\
\hline
\end{tabular}

${ }^{a}$ Total opportunities was determined by the number of participants in each experiment multiplied by the four critical items that each participant discussed. ${ }^{\mathrm{b}}$ Memory conformity refers to the total number of times that people reported the misinformation on the final independent memory test after being exposed to misleading information during the discussion. 


\section{Results and discussion}

Analyses

The primary goal of this research was to explore the associations between speaking order and (a) exposure to misinformation and (b) memory conformity. To address these questions, we analyzed the data at the level of each individual critical item, determining whether each person was exposed to misinformation, whether the person spoke first in discussing the item, and whether the person reported a false memory. A person was counted as speaking first in the discussion of a critical item if he or she was the first to provide some information in response to the discussion question. If the person was the first to speak, but did not provide any information (e.g., "I'm not sure"), this was not counted as speaking first.

Because each person discussed four critical items, items could not be treated as independent, but were nested within subjects for analysis. Additionally, because each person took part in the discussion with another person, responses could not be treated as independent, but were nested within dyads for analysis. Although the relationship between subjects and dyads was purely hierarchical (each subject was associated with only one dyad), the relationship between items and subjects was nonhierarchical (critical items were counterbalanced across conditions, so each subject was associated with four of the eight critical items). Our data set was therefore cross-classified (Goldstein, 1994).

To address the nested structure of the data, we analyzed each data set with multilevel mixed effects binary logistic regression, implemented through SPSS's generalized linear mixed models (GLMM: SPSS Version 19). We treated the three levels of data (critical items, subjects, and dyads) as categorical random effects. We treated speaking order as a categorical fixed effect with two levels (spoke first vs. spoke second). Whether each person was exposed to misinformation and whether that person was misled on a critical item were treated as binary outcomes (yes or no). In order to test the relationships between the fixed effect and the outcomes, GLMM used a binomial probability distribution for the outcomes and linked them to the fixed effect with a logit function. To address the cross-classifications in the data, the tests were conducted within the context of a hierarchical data structure in which each critical item was nested within subjects, and each subject was nested within dyads.

Speaking order and exposure to misinformation

Was speaking order associated with exposure to misinformation? Table 2 presents a cross-tabulation between speaking order and exposure to misinformation; we examined whether the trends were statistically reliable when dyads and subjects
Table 2 Speaking order for critical items in which people were exposed to misinformation, as percentages (raw numbers in parentheses)

\begin{tabular}{lll}
\hline Data Set & \multicolumn{2}{l}{ Speaking Order } \\
\cline { 2 - 3 } & First & Second \\
\hline Experiment 1 & $22.2(18)$ & $77.8(63)$ \\
Experiment 2 & $31.8(49)$ & $68.2(105)$ \\
Experiment 3 & $34.4(87)$ & $65.6(166)$ \\
Experiment 4 & $34.0(36)$ & $66.0(70)$ \\
Experiment 5 & $39.4(172)$ & $60.6(264)$ \\
\hline
\end{tabular}

were modeled as random effects. As Table 3 shows, for all five data sets, people who spoke second were more likely to be exposed to misinformation than were people who spoke first. As the odds ratios illustrate, these effects were relatively strong. For instance, in Experiment 4, people who spoke second were nearly nine times more likely to be exposed to misinformation than were those who spoke first. These findings fit with Lindsay's (2007) speculation that speaking order is fundamentally linked with exposure to misinformation: Across five experiments, people who initiated discussion had fewer opportunities to be misled because they were exposed to misinformation less often. A mini-meta-analysis using the random effect model (Borenstein, Hedges, Higgins, \& Rothstein, 2009) produced an aggregated odds ratio of 6.76 (95\% confidence interval [CI]: 4.99, 8.53; $p<.001)$ across the five experiments. This result demonstrates a strong relationship between speaking order and exposure to misinformation, such that people who spoke second were nearly seven times more likely to be exposed to misleading suggestions than were those who spoke first.

Speaking order and memory conformity

We now turn to our primary research question: Was speaking order associated with memory conformity? To eliminate exposure to misinformation as a potential confound, we examined only the critical items for which people were

Table 3 Associations between speaking order and exposure to misinformation for each data set

\begin{tabular}{lllllll}
\hline Data Set & $N$ cases $^{\mathrm{a}}$ & Coefficient & $\begin{array}{l}\text { Odds } \\
\text { Ratio }\end{array}$ & $95 \%$ CI & $p$ Value \\
\hline Experiment 1 & 160 & 1.80 & 6.04 & {$[2.99,12.16]$} & $<.001$ \\
Experiment 2 & 256 & 1.94 & 6.97 & {$[3.94,12.31]$} & $<.001$ \\
Experiment 3 & 384 & 2.06 & 7.86 & {$[4.69,13.14]$} & $<.001$ \\
Experiment 4 & 160 & 2.18 & 8.85 & {$[3.87,20.23]$} & $<.001$ \\
Experiment 5 & 640 & 1.43 & 4.16 & {$[2.86,6.07]$} & $<.001$ \\
\hline
\end{tabular}

${ }^{\text {a }} N$ cases was determined by the number of participants in each experiment multiplied by the four critical items that each participant discussed. 
exposed to misinformation. Table 4 presents a crosstabulation between speaking order and memory conformity; we examined whether these trends were statistically reliable when dyads and subjects were modeled as random effects. As Table 5 illustrates, in all five experiments, people who spoke second were more likely to be misled than were those who spoke first. This effect was significant for Experiments 1, 2, 3, and 5, and marginally significant for Experiment 4 . The odds ratios demonstrate that the association between speaking order and memory conformity was relatively strong. For instance, in Experiment 1, people who spoke second were over six times more likely to be misled than were those who spoke first. A mini-meta-analysis using the random-effects model produced an aggregated odds ratio of 3.60 (95\% CI: 2.31 , $4.90 ; p<.001)$ across the five experiments, demonstrating that people who spoke second were over three times more likely to be misled than were those who spoke first.

These results build on previous research by demonstrating an association between speaking order and memory conformity when people are actively involved in the conversation, not only when people observe others having a conversation (see Wright \& Carlucci, 2011). More to the point, these results also demonstrate an association between speaking order and memory conformity that cannot be caused by first speakers having less opportunity to be misled (see Gabbert et al., 2006; Lindsay, 2007). Taken together, the results show that when someone speaks first, they are much less likely to be exposed to misleading information, and when they are exposed to misinformation, they are much less likely to be misled than is someone who does not begin the conversation.

\section{Rival hypotheses}

We next tested a number of plausible alternative explanations for the apparent relationship between speaking order and memory conformity.

Baseline memory confidence and accuracy To examine the possibility that people who have better memories, or people

Table 4 Critical items in which people exhibited memory conformity, given as percentages of the number of opportunities they had to be misled (raw number of misled items/raw number of opportunities to be misled), as a function of speaking order and data set

\begin{tabular}{lll}
\hline Data Set & \multicolumn{2}{l}{ Speaking Order } \\
\cline { 2 - 3 } & First & Second \\
\hline Experiment 1 & $33.3(6 / 18)$ & $76.2(48 / 63)$ \\
Experiment 2 & $35.0(17 / 49)$ & $66.7(70 / 105)$ \\
Experiment 3 & $27.6(24 / 87)$ & $57.2(95 / 166)$ \\
Experiment 4 & $33.3(12 / 36)$ & $54.3(38 / 70)$ \\
Experiment 5 & $31.4(54 / 172)$ & $48.1(127 / 264)$ \\
\hline
\end{tabular}

Table 5 Associations between speaking order and memory conformity for each data set

\begin{tabular}{lllllll}
\hline Data Set & $N$ cases $^{\mathrm{a}}$ & Coefficient & $\begin{array}{l}\text { Odds } \\
\text { Ratio }\end{array}$ & $95 \%$ CI & $p$ Value \\
\hline Experiment 1 & 81 & 1.90 & 6.66 & {$[2.02,21.97]$} & .002 \\
Experiment 2 & 154 & 1.39 & 4.00 & {$[1.85,8.66]$} & .001 \\
Experiment 3 & 253 & 1.25 & 3.49 & {$[1.98,6.15]$} & $<.001$ \\
Experiment 4 & 106 & 0.81 & 2.23 & {$[0.95,5.34]$} & .064 \\
Experiment 5 & 436 & 0.68 & 1.97 & {$[1.31,2.96]$} & .001 \\
\hline
\end{tabular}

${ }^{\mathrm{a}} N$ cases was determined by the number of critical items for which each participant was exposed to misinformation during discussion.

who are more confident in their memories, tend to speak first and also tend to be less influenced by others' suggestions, we examined data for the four critical items that were not discussed to calculate an overall baseline memory accuracy score and an overall baseline memory confidence score for each person. We then calculated the total number of times that each person spoke first when discussing critical details and examined whether a higher tendency to speak first was associated with higher baseline memory accuracy or confidence. As Table 6 shows, speaking order was not related to memory accuracy in any experiment, and in four of the five experiments, speaking order was not related to baseline memory confidence, suggesting that neither baseline memory accuracy nor memory confidence can fully account for the association between speaking order and memory conformity.

In Experiment 3, we found a significant relationship between speaking order and memory confidence, such that people who were more confident were also more likely to speak first. Given that ten separate statistical tests were conducted, there is a reasonable possibility (a 50:50 chance) that this one significant result is simply due to a Type I error (Lehmann \& Romano, 2005). However, in Experiment 3, people were led to believe that they had the same, better, or worse ability to see the movie than their discussion partner. The results showed that people who were led to believe that

Table 6 Correlations between speaking order and baseline memory accuracy and confidence

\begin{tabular}{lll}
\hline Data Set & $\begin{array}{l}\text { Baseline Memory } \\
\text { Accuracy }\end{array}$ & $\begin{array}{l}\text { Baseline Memory } \\
\text { Confidence }\end{array}$ \\
\hline Experiment 1 $(N=40)$ & $r=.034, p=.833$ & $r=-.127, p=.435$ \\
Experiment 2 $(N=64)$ & $r=-.091, p=.473$ & $r=.174, p=.173$ \\
Experiment 3 $(N=96)$ & $r=.025, p=.809$ & $r=.227, p=.026^{*}$ \\
Experiment 4 $(N=42)$ & $r=.231, p=.140$ & $r=.129, p=.416$ \\
Experiment 5 $(N=160)$ & $r=-.045, p=.570$ & $r=-.004, p=.964$ \\
Experiment 3 control & $r=.042, p=.846$ & $r=-.072, p=.739$ \\
only $(N=24)$ & & \\
\hline${ }^{*} p<.05$ & &
\end{tabular}


they were superior to their partners were more confident in their memories (despite being no more accurate), and were also more likely to speak first than were people in the other conditions. When we repeated the analyses using only the control (same-ability) condition in Experiment 3, we found no relationship between speaking order and either memory accuracy $(r=.04, p=.85)$ or memory confidence $(r=-.07$, $p=.74)$, supporting the idea that the experimental manipulation of credibility affected both speaking order and confidence. Although confidence and speaking order might be affected in a similar way by different circumstances (such as the manipulation in Exp. 3), there was no evidence of a consistent relationship between the two variables, and as such, it is not a compelling explanation for the association between speaking order and memory conformity.

Speaker credibility The finding that the relationship between speaking order and memory confidence was affected by the speaker's credibility highlights the possibility that the relationship between speaking order and memory conformity might also be affected by the speaker's credibility. For instance, it might be that speakers who are more credible are more likely to speak first, less likely to be exposed to misinformation, and less likely to be misled. Given that in Experiments 3 and 5 we manipulated speaker credibility, and Experiment 2 included romantic couples who might have existing beliefs about each other's credibility, we reanalyzed the data using only the control conditions for these experiments. The same results held for the control conditions in all three experiments (see Table 7). In addition, the same pattern of results held for Experiments 1 and 4 , in which speaker credibility was not manipulated. Taken together, these results suggest that the relationship between speaking order and memory conformity cannot be explained by speaker credibility.

Dispute Another explanation might be that first speakers are less susceptible to memory conformity only when the other

Table 7 Associations between speaking order and exposure to misinformation, and between speaking order and memory conformity for each data set (control trials only)

\begin{tabular}{lllll}
\hline Data Set $\quad N$ Items & Coefficient & $\begin{array}{l}\text { Odds } \\
\text { Ratio }\end{array}$ & $95 \%$ CI & $p$ Value
\end{tabular}

Exposure to Misinformation

\begin{tabular}{rlllll} 
Experiment 2 & 128 & 1.74 & 5.70 & {$[2.56,12.69]$} & $<.001$ \\
Experiment 3 & 96 & 2.12 & 8.22 & {$[2.99,22.55]$} & $<.001$ \\
Experiment 5 & 160 & 1.83 & 6.28 & {$[2.67,14.80]$} & $<.001$ \\
Memory Conformity & & & & \\
Experiment 2 & 78 & 1.19 & 3.30 & {$[1.14,9.55]$} & .028 \\
Experiment 3 & 61 & 1.51 & 4.54 & {$[1.14,17.99]$} & .032 \\
Experiment 5 & 112 & 0.92 & 2.51 & {$[1.04,6.08]$} & .041 \\
\hline
\end{tabular}

person fails to contradict what they say. We tested the hypothesis that dispute between speakers moderates the relationship between speaking order and memory conformity and found no support for it: Dispute (yes or no) did not moderate the relationship between speaking order and memory conformity (Exps. 1-4, $p \mathrm{~s}>.3$; Exp. 5, $p>.05$ ).

Prior agreement Finally, it might be that second speakers are more likely to be misled than are first speakers because they are also more likely to agree to use the misinformation as an answer during the discussion (whether they genuinely believe it or not), and then they simply use the same answer that they had previously agreed to (prior agreement) on the individual memory test. In fact, in all five experiments, the relationship between speaking order and memory conformity no longer held when prior agreement was controlled (Exps. 1, 4, and 5, $p \mathrm{~s}>.1$; Exps. 2 and 3, $p \mathrm{~s}>.05$ ). In other words, prior agreement is a better predictor of memory conformity than is speaking order. However, prior agreement was closely associated with speaking order $(\phi=.257, p<.001)$, and given that speaking order must logically precede prior agreement, prior agreement cannot affect speaking order. Instead, we argue that speaking order affects prior agreement, such that people who speak second are more likely to agree with the first speaker's suggestions during discussion than vice versa. As such, this confound is not fatal for our hypotheses, but instead suggests a possible mechanism through which speaking order might affect memory conformity: by influencing the likelihood that people will agree with information provided in a social setting.

\section{Explanations of the relationship}

These findings are consistent with the literature regarding social influence, cognitive dissonance, persuasion techniques, and the effects of anchoring and primacy on persuasion, which all lend support to the idea that information encountered first may be more influential than is subsequent information. But our analyses do not provide any evidence of causation or allow us to differentiate between these possible mechanisms. Further investigation is thus warranted, to identify the specific processes responsible for the association between speaking order and memory conformity and the explanations that best account for the association.

Ideally, this research should aim to tease apart the contributions of different underlying processes to further our understanding of memory conformity (Wright et al., 2009). As we have noted, the association could be accounted for by a variety of different processes, including an interpersonal metacognitive heuristic, informational influence, normative and self-normative influence, or cognitive dissonance reduction. Of course, the association may not be causal: It might 
be that people who are more extraverted tend to speak first and also tend to be less influenced by others' suggestions. But Wright and Carlucci's (2011) research demonstrating that people who observe conversations are also more influenced by first speakers suggests that the association is indeed causal, and not due to interpersonal interactions between the speakers in a conversation. Wright and Carlucci's order effects lend support to the idea that the association is due to the primacy of the first speaker's information, rather than to another underlying social process. One possible explanation of this primacy effect is that people rely on a metacognitive heuristic that the first speaker is most likely to be accurate, and they fail to discount the rule even when the speaking order is out of the speakers' control (Wright \& Carlucci, 2011).

\section{Memory conformity in everyday life}

In a practical sense, the relationship between speaking order and memory conformity may be even more powerful than has been identified in this and other research; our results suggest that in everyday life, people who do not speak first are not only more likely to encounter opportunities to be misled, but when they do encounter these opportunities, they are more likely to be influenced. By controlling for exposure to misinformation, researchers may substantially underestimate the true effect of speaking order on memory conformity in everyday life.

It seems likely that speaking order interacts with other features of the social interaction to produce memory conformity. Discussion features such as the relationship between discussion partners and their perceptions of each other's credibility affect who will most likely be influenced by misinformation (e.g., Allan et al., 2011; French et al., 2008, 2011; Hope, Ost, Gabbert, Healey, \& Lenton, 2008); perhaps these situational features also affect who is most likely to speak first. Additionally, it may be that response order is important in some social interactions but overridden in others. For instance, it would be interesting to pit speaking order against speaker confidence and to compare a speaker who speaks confidently with a speaker who appears to doubt his or her own words. Future investigation of these types of interactions will help to further elucidate the roles that different factors play in producing memory conformity.

Processes underlying memory conformity

In this research, we investigated the effect of exposure to false information on later memory reports; the use of false information and the focus on memory errors was a deliberate attempt to maintain experimental control of the processes under investigation. However, the processes underlying memory conformity are likely to be adaptive and to produce outcomes that are typically beneficial by enabling people to absorb extra information from different sources, to extend their knowledge, and to create a shared understanding with other people (French, Garry, \& Loftus, 2009; French et al., 2008, 2011; Hardin \& Higgins, 1996; Jaeger, Lauris, Selmeczy, \& Dobbins, 2011; Meade \& Roediger, 2002). Speaking order may play a role in this process by providing an accessible cue that speaks to the likely accuracy of shared information; as a result, people may be better able to monitor shared information, rejecting information when it is unlikely to be accurate and relying on the information when it is likely to be accurate. Of course, in situations in which we rely on people's memory reports to inform consequential decisions, there are potentially significant costs when someone makes an error and shares the inaccurate information with other people; our findings suggest that this situation may be especially dangerous if the error is shared before other people have had a chance to share their own memories. However, in drawing conclusions from memory conformity research, it is important that researchers consider not only the practical implications of memory errors, but also the theoretical implications for the social and memorial processes implicated in both accurate and inaccurate memory conformity.

\section{Summary and conclusion}

Taken together, the results of these exploratory analyses provide evidence of a strong association between speaking order and memory conformity, and they suggest that this relationship may be even stronger in everyday situations when other related factors are at play. Further investigation will be required in order to shed light on the exact nature of the relationship between speaking order and memory conformity, to differentiate the social and memorial processes underlying memory conformity, and to examine how various social factors contribute to and interact with speaking order and memory conformity.

Author Note This research was partly funded by a grant from the Curtin University School of Psychology and Speech Pathology Research Allocation Fund. Correspondence concerning this article should be addressed to Lauren Hewitt, School of Psychology and Speech Pathology, Curtin University, Perth, Western Australia, 6845. Phone: +61 892663450 Email: lauren.hewitt@curtin.edu.au

\section{References}

Allan, K., Midjord, P., Martin, D., \& Gabbert, F. (2011). Memory conformity and the perceived accuracy of self versus other. Memory \& Cognition, 40, 280-286. doi:10.3758/s13421-011-0141-9

Asch, S. E. (1952). Social psychology (pp. 450-501). NY: Prentice Hall. Borenstein, M., Hedges, L., Higgins, J. P. T., \& Rothstein, H. R. (2009). Introduction to meta-analysis. West Sussex, UK: Wiley. 
Cialdini, R. B., \& Goldstein, N. J. (2004). Social influence: Compliance and conformity. Annual Review of Psychology, 55, 591-621. doi:10.1146/annurev.psych.55.090902.142015

Deutsch, M., \& Gerard, H. B. (1955). A study of normative and informational social influences upon individual judgment. Journal of Abnormal and Social Psychology, 51, 629-636. doi: $10.1037 / \mathrm{h} 0046408$

Englich, B., Mussweiler, T., \& Strack, F. (2005). The last word in courtA hidden disadvantage for the defense. Law and Human Behavior, 29, 705-722. doi:10.1007/s10979-005-8380-7

Festinger, L., \& Carlsmith, J. M. (1957). Cognitive consequences of forced compliance. Journal of Abnormal and Social Psychology, 58, 203-210. doi: $10.1037 / \mathrm{h} 0041593$

French, L. (2008). Social aspects of discussion affect capitulation and susceptibility to false memories (Doctoral thesis, Victoria University of Wellington, Wellington, New Zealand). Retrieved from http://researcharchive.vuw.ac.nz//handle/10063/499

French, L., Garry, M., \& Loftus, E. F. (2009). False memories: A kind of confabulation in non-clinical subjects. In W. Hirstein (Ed.), Confabulation: Views from neuroscience, psychiatry, psychology, neurology, and philosophy (pp. 33-66). Oxford, UK: Oxford University Press.

French, L., Garry, M., \& Mori, K. (2008). You say tomato? Collaborative remembering between intimate couples leads to more false memories than collaborative remembering between strangers. Memory, 16, 262-273. doi:10.1080/09658210701801491

French, L., Garry, M., \& Mori, K. (2011). Relative - not absolutejudgments of credibility affect susceptibility to misinformation conveyed during discussion. Acta Psychologica, 136, 119-128. doi:10.1016/j.actpsy.2010.10.009

Gabbert, F., Memon, A., \& Wright, D. B. (2006). Memory conformity: Disentangling the steps towards influence during a discussion. Psychonomic Bulletin \& Review, 13, 480-485. doi:10.3758/ BF03193873

Garry, M., French, L., Kinzett, T., \& Mori, K. (2008). Eyewitness memory following discussion: Using the Mori technique with a Western sample. Applied Cognitive Psychology, 22, 431-439. doi:10.1002/acp. 1376

Goldstein, H. (1994). Multilevel cross-classified models. Sociological Methods and Research, 22, 364-3775. doi:10.1177/ 0049124194022003005

Hardin, C. D., \& Higgins, E. T. (1996). Shared reality: How social verification makes the subjective objective. In R. M. Sorrentino \& E. T. Higgins (Eds.), Handbook of motivation and cognition (The interpersonal context, Vol. 3, pp. 29-84). New York, NY: Guilford Press.

Hoffman, H. G., Granhag, P. A., Kwong See, S. T., \& Loftus, E. F. (2001). Social influences on reality-monitoring decisions. Memory \& Cognition, 29, 394-404. doi:10.3758/BF03196390
Hope, L., Ost, J., Gabbert, F., Healey, S., \& Lenton, E. (2008). "With a little help from my friends..".: The role of co-witness relationship in susceptibility to misinformation. Acta Psychologica, 127, 476484. doi:10.1016/j.actpsy.2007.08.010

Jaeger, A., Lauris, P., Selmeczy, D., \& Dobbins, I. G. (2011). The costs and benefits of memory conformity. Memory \& Cognition, 40, 101-112. doi:10.3758/s13421-011-0130-Z

Lehmann, E. L., \& Romano, J. P. (2005). Generalizations of the family-wise error rate. The Annals of Statistics, 33, 1138-1154. doi:10.1214/09-AOS734

Lindsay, D. S. (2007). Order effects in collaborative memory contamination? Comment on Gabbert, Memon, and Wright (2006). Psychonomic Bulletin \& Review, 14, 1010. doi:10.3758/BF03194137

Lindsay, D. S. (2008). Source monitoring. In H. L. Roediger III (Vol. Ed.) \& J. Byrne (Series Ed.), Cognitive psychology of memory: Vol. 2. Learning and memory: A comprehensive reference (pp. 325-348). Oxford, UK: Elsevier.

Meade, M. L., \& Roediger, H. L., III. (2002). Explorations in the social contagion of memory. Memory \& Cognition, 30, 995-1009. doi:10.3758/BF03194318

Miller, N., \& Campbell, D. T. (1959). Recency and primacy in persuasion as a function of the timing of speeches and measurements. Journal of Abnormal Psychology, 59, 1-9. doi: $10.1037 / \mathrm{h} 0049330$

Mori, K. (2003). Surreptitiously projecting different movies to two subsets of viewers. Behavior Research Methods, Instruments, \& Computers, 35, 599-604. doi:10.3758/BF03195539

Mori, K., \& Arai, M. (2010). No need to fake it: Reproduction of the Asch experiment without confederates. International Journal of Psychology, 45, 390-397. doi:10.1080/00207591003774485

Sherif, M. (1937). An experimental approach to the study of attitudes. Sociometry, 1, 90-98. doi:10.2307/2785261

Tavris, C., \& Aronson, E. (2007). Mistakes were made (but not by me). London, UK: Pinter \& Martin.

Wright, D. B., \& Carlucci, M. E. (2011). The response order effect: People believe the first person who remembers an event. Psychonomic Bulletin \& Review, 18, 805-812. doi:10.3758/ s13423-011-0089-6

Wright, D. B., Memon, A., Skagerberg, E. M., \& Gabbert, F. (2009). When eyewitnesses talk. Current Directions in Psychological Science, 18, 174-178. doi:10.1111/j.1467-8721.2009.01631.x

Wright, D. B., Self, G., \& Justice, C. (2000). Memory conformity: Exploring misinformation effects when presented by another person. British Journal of Psychology, 91, 189-202. doi:10.1348/ 000712600161781

Wright, D. B., \& Villalba, D. K. (2012). Memory conformity affects inaccurate memories more than accurate memories. Memory, 20 , 254-265. doi:10.1080/09658211.2012.654798 